TM-1671

[SSCL-N-731]

\title{
SSC Dipole Magnet Measurement and Alignment Using Laser Technology
}

A. Lipski, J. A. Carson and W. F. Robotham

Fermi National Accelerator Laboratory

P.O. Box 500

Batavia, Illinois 60510

June 1990

* Presented at the 1990 International Industrial Symposium on the Super Collider (IISSC), Miami Beach, Florida, March 14-16, 1990. 


\title{
SSC DIPOLE MAGNET MEASUREMENT AND ALIGNMENT USING LASER TECHNOLOGY
}

\author{
A. Lipski, J.A. Carson and W.F. Robotham \\ Fermilab National Accelerator Laboratory \\ Box 500 \\ Batavia, IL 60510
}

\begin{abstract}
Advancing into the prototype production stage of the SSC dipole magnets has introduced the need for a reliable, readily available, accurate alignment measuring system which gives results in real time. Components and subassemblies such as the cold mass and vacuum vessel are being measured for various geometric conditions such as straightness and twist. Variations from nominal dimensions are also being recorded so they can be compensated for during the final assembly process. Precision laser alignment takes specific advantages of the properties of helium-neon laser. The laser beam forms a straight line of the greatest accuracy. When combined with an optically produced perpendicular plane, this results in a system of geometric references of unparalleled accuracy. This paper describes the geometric requirements for SSC dipole magnet components, sub and final assemblies as well as the use of laser technology for surveying as part of the assembly process.
\end{abstract}

\section{INTRODUCTION}

The laser alignment system will replace the present optical alignment process. The laser system offers the following advantages:

- Results are in real time

- Eliminates the human factor

- Reduces survey time

- Eliminates the need for a special survey crew

- Repeated measurements for time dependent variations can be obtained with minimal preparations

- Hard copy of results (numerically and graphically) can be obtained at any time 
The laser system itself is commercially available from Hamar Laser which will be applied to the alignment procedure. The term "alignment procedure" is defined as the method to obtain a finished magnet which has the magnetic centerline and vertical plane aligned, within specified tolerances to some datum located on the outside of the vacuum vessel. (Most likely - bottom of vessel foot and a fiducial located on foot.) The alignment procedure excludes the alignment of the interconnect region at the magnet ends. In addition, this procedure does not attempt to compensate for inherent errors built into the cold mass assembly, namely the cold mass straightness between its supports is unreconcilable as are variations of the magnetic vertical plane. Only average values ( $x$; $y)$ of the beam tube centerline taken in specified locations along the length of the cold mass and those of the magnetic vertical plane are as reference used in the alignment procedure. Note that due to the crude tolerances of the drawn beam tube its reference center line will be a result of best fitted curve.

For budgetary considerations the adopted construction philosophy of the cryostat has been to minimize the need for tight tolerances in piece parts and assemblies. It is also realized that design of stacked up parts results in tolerance build up which can affect magnet alignment. Part of the alignment process is taking into account the dimensional variance of parts from their nominal dimensions and compensates for it during the assembly process (Figure 1). This part, however, is not going to be covered under the scope of this paper. This paper will only deal with the portion where laser technology is replacing commonly used optical survey instrumentation. The various steps which include laser alignment of cold mass vacuum vessel and the final assembly will be briefly discussed and illustrated (see Appendices).

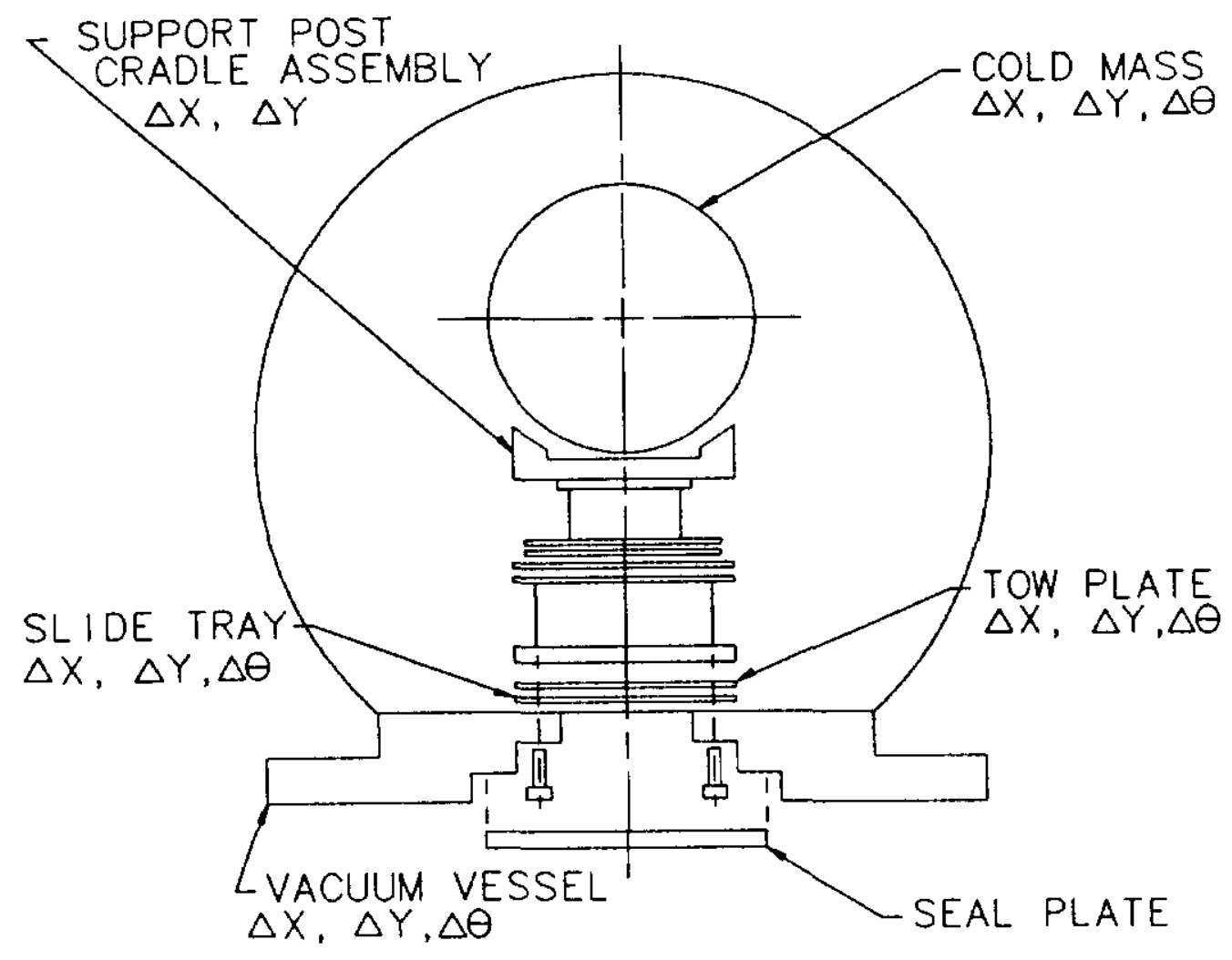

Figure 1. Components Which Affect Alignment 


\section{WHAT IS LASER ALIGNMENT}

Precision laser alignment takes specific advantages of the properties of a heliumneon laser. This type of laser produces an intense beam of red light which is a straight line of the greatest accuracy. When bent through a precise $90^{\circ}$ angle by a special rotating penta prism a very flat plane is produced. The combination of the straight line and the flat plane results in a laser equivalent of a surface plate, straight edge, cylindrical square, etc. It is a system of geometric references of great accuracy. The laser can be precisely leveled to produce a unique and versatile alignment system.

\section{COLD MASS}

\section{Tooling Alignment}

Prior to the placement of the cold mass on its support tooling it is essential to align all 5 centers of the support tooling with the survey line of sight. The concept used here is to establish the laser beam as the centerline between support tooling \#1 and \#5 and aligning the rest of the supports centers with the previously established centerline. The 4-axis bore target measuring both the two axis centering and squareness (pitch and yaw) is being held by an adjustable target holder which is supported by two micrometer stick legs and one spring loaded plunger leg (Appendix 1).

\section{Straightness - Horizontal and Vertical}

The incorporation of precision skin allows for straightness measurement at any increment along the length of the cold mass. As a first step a survey of the horizontal straightness is taken. The equipment used here will consist of the 4-axis target, the single axis sweep target and the remote scan optical square. The laser unit is mounted in $a$ vertical position on a fixture. The beam which is emitted from the center top laser head is "bucked-in" with the theoretical beam tube centers in both ends of the cold mass using the 4axis bore target. By turning the thumb lever on the laser head the beam is now emitted from the side of the laser head and parallel to the top of the alignment bar to the center of the remote scan optical square. The vertical sweep plane which is produced will be parallel to the nominal cold mass centerline and perpendicular to the horizontal beam aimed at the remote scan optical square. Moving a single axis sweep target along the cold mass length would indicate the variation of the skin from straightness with respect to the referenced centerline.

Similarly for vertical measurement of straightness the laser is left in its place while the remote scan optical square is moved to a horizontal position right above the laser unit. This will cause the remote scan optical square to sweep a plane which is parallel to the nominal centerline and perpendicular to the beam aimed toward the remote scan unit. Rotation of $180^{\circ}$ of the cold mass and repetition of the survey sequence would indicate the effect of gravity on the results (Appendices 2 and 3 ).

\section{Twist Measurement}

As part of the cold mass survey it is important to study the level of twist that has been introduced to the cold mass during its assembly process. The amount of twist will effect the establishment of the vertical plane later on in the process (not within the scope of this paper). Cold mass twist together with deviations of vertical plane measurements coupled with angular deviations of vacuum vessel (Figure 2) and slide tray are being compensated for by rotating out the cold mass prior to being anchored to the center support post (not covered in this paper). 




Figure 2. Vacuum Vessel Deviations

The twist measurement utilizes the laser's scanning capability, thus, forming a horizontal plane. Two single axis sweep targets are placed on both ends of the horizontal surface of a twist fixture. This fixture is locked into the press fit " $\mathrm{V}$ " keys which are accurately positioned on either side of the cold mass. Any twist in the cold mass will be translated into an angular displacement which will be read by the targets placed on the fixture. Twist measurements are taken in the five post center line locations (Appendix 4).

\section{Define Geometric Center}

A target is installed in the beam tube and moved longitudinally. The target center position is measured with respect to the established sight line and $\Delta x, \Delta y$ values are recorded. This procedure is repeated at 24 longitudinal locations as follow:

a. At each support

b. Magnet ends

c. 3 equally spaced positions between supports or supports and ends

This data will be used to define the center of the magnet in the finished assembly as well as measure the straightness and concentricity of the beam tube with respect to the cold mass. The laser target is mounted on the centerline of an air bearing/target transport assembly which is connected to the end of a self propelled in-tube shuttle (inchworm) via double spherical air bearing. The air-bearing/target transport assembly will center itself in the beam tube and will maintain its angular positioning due to the counterweight located on its lower level while the double spherical air bearing will prevent any angular input from the inchworm to the air-bearing/target transport assembly (Appendix 5). 


\section{VACUUM VESSEL}

\section{Tooling Supoorts Alignment}

Prior to the alignment of the vacuum vessel it is essential to align its tooling supports. In order to compensate for the possible effects of the combined "dead weight" of vacuum vessel and posts on the tooling supports, those are being simulated via an empty vacuum vessel and a suspended "dummy" cold mass which are loading the tooling supports during the alignment process.

The initial alignment will take place before the vacuum vessel is moved into place, yet the final alignment will be done with the vacuum vessel on top of the tooling supports. The laser unit will be in its scanning mode, thus, forming a horizontal plane. The two top support plates are being leveled and elevated to form a horizontal plane using three single axis sweep targets at which time they are locked in place (Appendix 6).

\section{Level Survey of Support Surfaces}

The vacuum vessel subassembly, although made to exacting tolerances derived from assembly fixturing, is expected to have geometric variations induced primarily by welding stresses. These variations from the nominal dimensions must be measured so that they could be compensated for during the final assembly of the magnet.

The critical geometric surfaces of the vacuum vessel are the surfaces to which the five support posts will be attached. These surfaces must be measured and deviations from the nominal condition determined. The bottom of the two vacuum vessel support feet serves as the horizontal reference plane while the center of the fiducial holes within the support feet forms the vertical reference plane. The vacuum vessel feet are bolted to a plane tooling surface and "dead weight" load applied to simulate cold mass and supports the weight. Deviation from the ideal geometry are measured at the five support surfaces, thus, establishing a $\Delta y$ and $\Delta \theta$ for each support location (Figure 2).

The laser is at a scanning mode, thus, forming a leveled horizontal plane. The two single axis sweep targets placed on both ends of the support pad centerline, which is perpendicular to the vacuum vessel axis, will indicate the $\Delta y$ deviation from nominal (Appendix 7).

\section{FINAL ASSEMBLY}

\section{Define Nominal Location of Cold Mass in Vacuum Vessel}

At this time it is necessary to establish the survey sight line which coincides with the nominal magnet center with respect to the magnet's horizontal and vertical reference planes (Figures 3). Since it will be very impractical to form a target which will be referenced from the external reference planes (namely the bottom of the vacuum vessel support feet and the fiducial hole within the support feet) it will be assumed that the hole in the support pad within the vacuum vessel was machined per specifications and thus it will serve as datum. A 4-axis target mounted in a fixture and located in the support pad hole over the support tooling will be used to "buck in" the laser beam to where it will form the survey sight line, at which time the laser location will be registered. This location will be used in a later stage to verify the magnet's final position and determine the assembly errors (Appendix 8). 


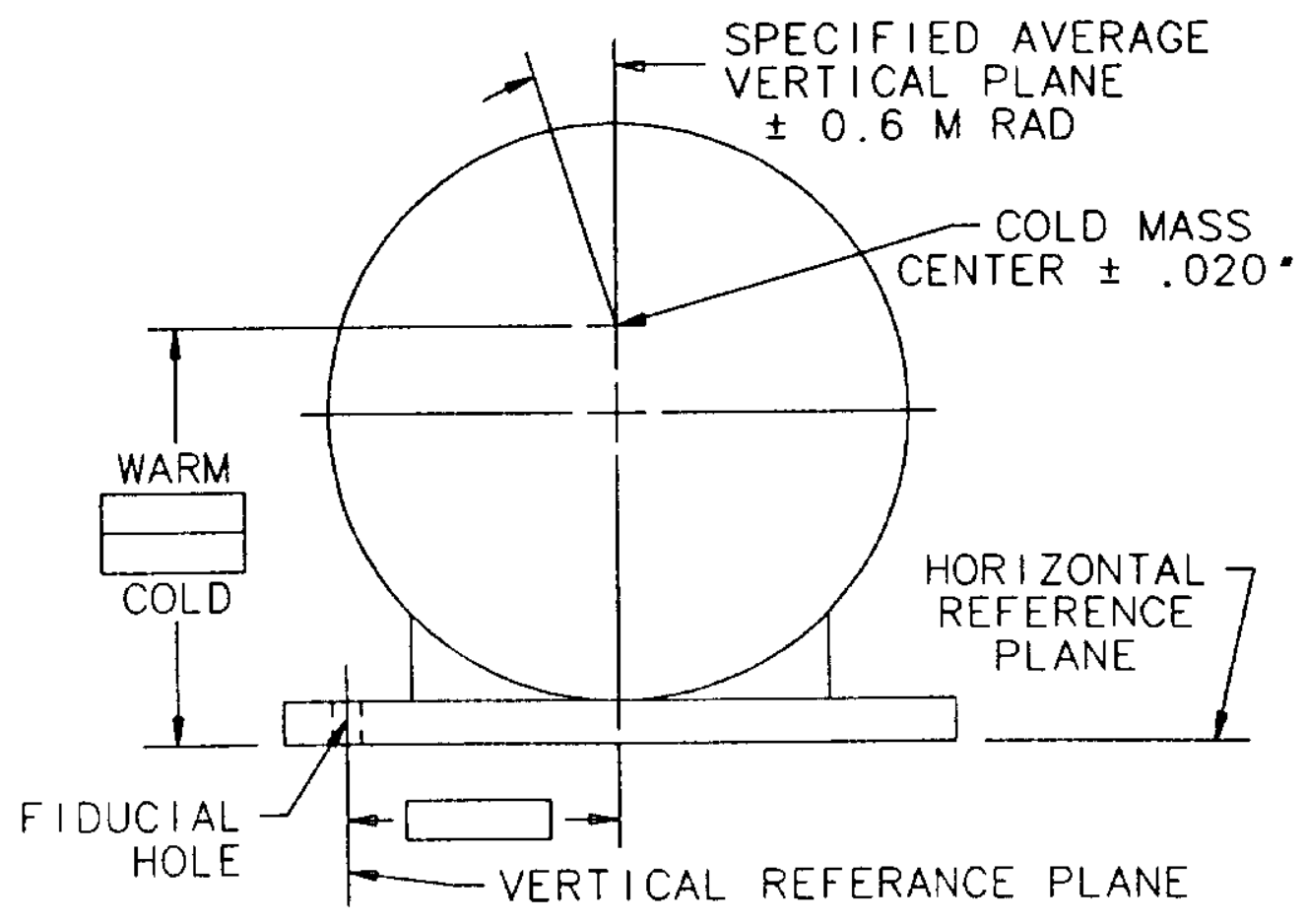

Figure 3. SSC Dipole Magnet Geometric Requirements

\section{Define Reference Plane for Suppert Posts Alignment}

After the cold mass/cryostat assembly has been placed in the vacuum vessel, plugs are inserted through access holes in the vacuum vessel and into a hole located in the bottom of each support post. Using a fixture, the plugs are then centered in the support pad hole. The lower portion of each plug has a flat surface machined in it to accommodate the base of a single axis sweep target. The laser beam, via a remote scan optical square, forms a vertically sweeping plane. Most of the alignment will consist of merely rotating the plug and target to its extreme position and then aligning the target in place with the rest of the plugs, thus, forming a reference plane from which the adjustment of each plug in the " $x$ " direction will be initiated.

The variations of all the components which effect the " $x$ " axis position as well as the sagitta values added to or subtracted from the nominal position with respect to the magnet's vertical datum plane will determine the final location of the plugs in the " $x$ " direction (Figures 4 and 5). The posts are then secured to the vacuum vessel (Appendix 9).

\section{Cenfirmation}

To confirm the geometric center a target is reinserted into the beam tube and readings taken at the five support locations. In order to do this, the laser unit is brought back to its registered location previously established, using the same floating 4-axis target (Appendix 8). Deviations from nominal are expected to be within the given tolerance range. Deviations which exceed the range are recognized as assembly errors. Some adjustment can be done to correct these errors. Corrections along the " $x$ " axis can be easily accomplished by loosening the appropriate support post from the vacuum vessel and repositioning it. Errors in the " $y$ " axis are more difficult to correct since these require jacking the post up and adding or eliminating shims through the access hole in the bottom 


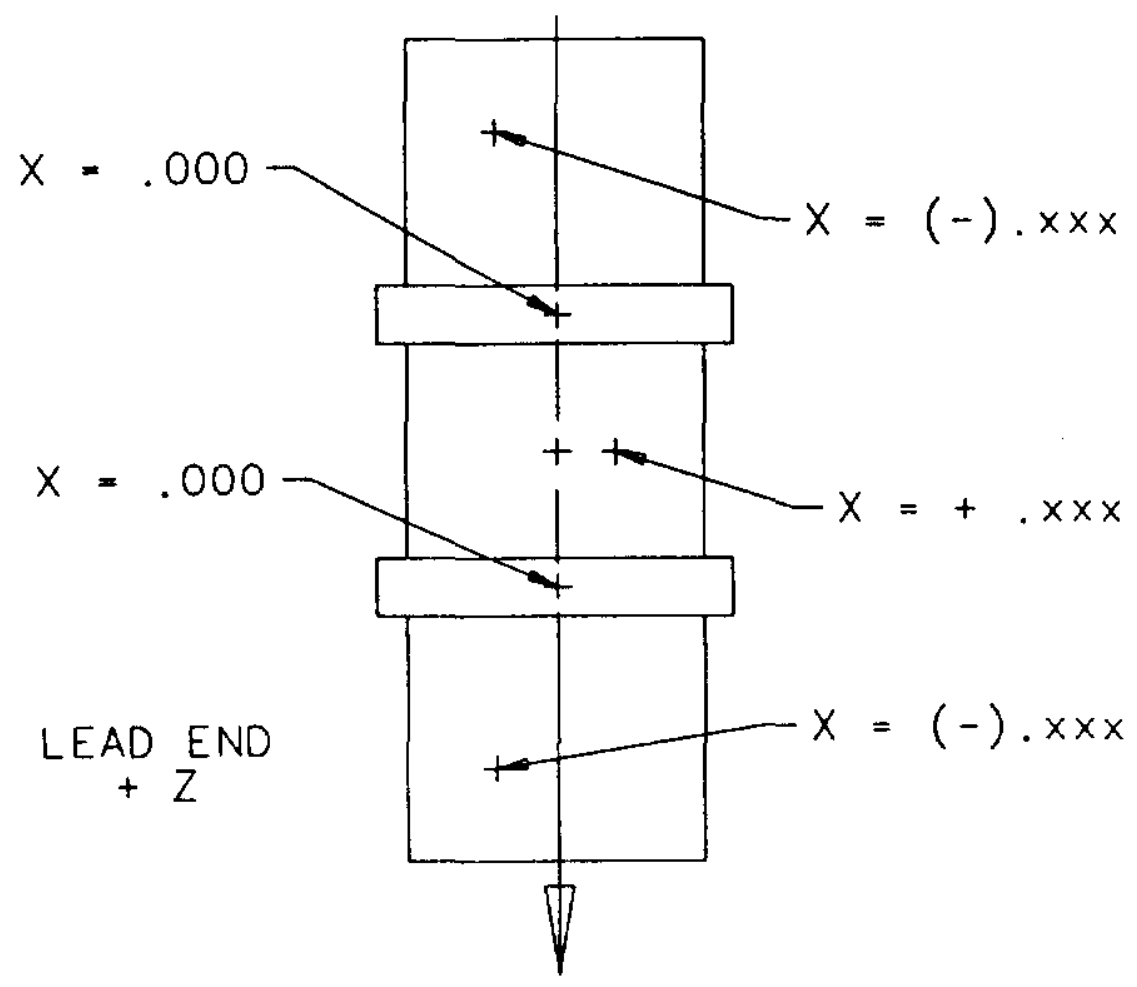

Figure 4. Plan View of



Figure 5. Setting Center on X 
of the vacuum vessel in addition to the loosening of the support post. Vertical plane errors requires disassembly to correct (Appendix 10).

\section{ALIGNMENT ERRORS}

Possible alignment errors can result from two sources:

a. Measurement errors of component parts and assembly process

b. Plastic deformation of components occurring after the final assembly was completed

\section{Measurement Errors}

Applying laser technology to the alignment process has the potential to substantially reduce measurement errors. The red beam from the laser is always visible, consequently, alignment is simplified and the possibility of error minimized. The twoaxis readout or the computer screen display geometric errors directly thus, eliminating "eyeball" mistakes. In a short time operators can be trained to use the system and perform tasks which would normally be considered for experts only. In addition the dynamic testing" allows the operator to have the readout continually displaying the horizontal and vertical variations from the nominal line or plane and make corrections instantaneously. Table 1 denotes the contribution of the various elements to the final assembly total tolerances.

Table 1. Alignment Error Resulting from Components and Assemblies

Obtainable Measurement Accuracy

\begin{tabular}{|c|c|c|c|c|}
\hline Component & $x($ in) & $Y$ Y(in) & Q(mrad) & $\begin{array}{l}\text { Laser } \\
\text { Alignment }\end{array}$ \\
\hline Average cold mass & \pm .002 & \pm .002 & \pm 0.1 & Yes \\
\hline Cradle/support assy & \pm .001 & \pm .001 & - & No \\
\hline Tow Plate & \pm .001 & \pm .001 & \pm 0.1 & No \\
\hline Slide tray & \pm .001 & \pm .001 & \pm 0.1 & No \\
\hline Vacuum vessel & \pm .005 & \pm .002 & \pm 0.1 & Yes \\
\hline Assembly & \pm .002 & \pm .002 & \pm 0.05 & Yes \\
\hline Total (RMS)* & \pm .0024 & \pm .0016 & \pm 092 & \\
\hline
\end{tabular}

* Root mean square 


\section{Plastic Deformation Errors}

Alignment errors resulting from plastic deformation involve creep of the cradle/support assembly and stress relaxation of the welded vacuum vessel assembly. These values need to be determined. In addition, handling, storage, and shipping of a magnet can possibly cause the alignment to be disturbed. The physical limits that can be imposed on a magnet (i.e. handling loads, storage temperatures, etc.) will have to be established.

\section{SUMMARY}

A considerable effort will be required to develop and demonstrate the alignment accuracy put forth in this paper. Formalization of procedures, gaining experience using the new laser technology, measurement fixtures and a determination of component stability must all be addressed. The goal of this paper was to offer a different approach to alignment via laser technology which promises to be faster, more accurate, reliable and simplified yet practical in a production environment.

\section{ACKNOWLEDGEMENTS}

The authors gratefully acknowledge the sincere contribution and professional performance of $R$. Dixon and his team as well as $K$. Swanson. 

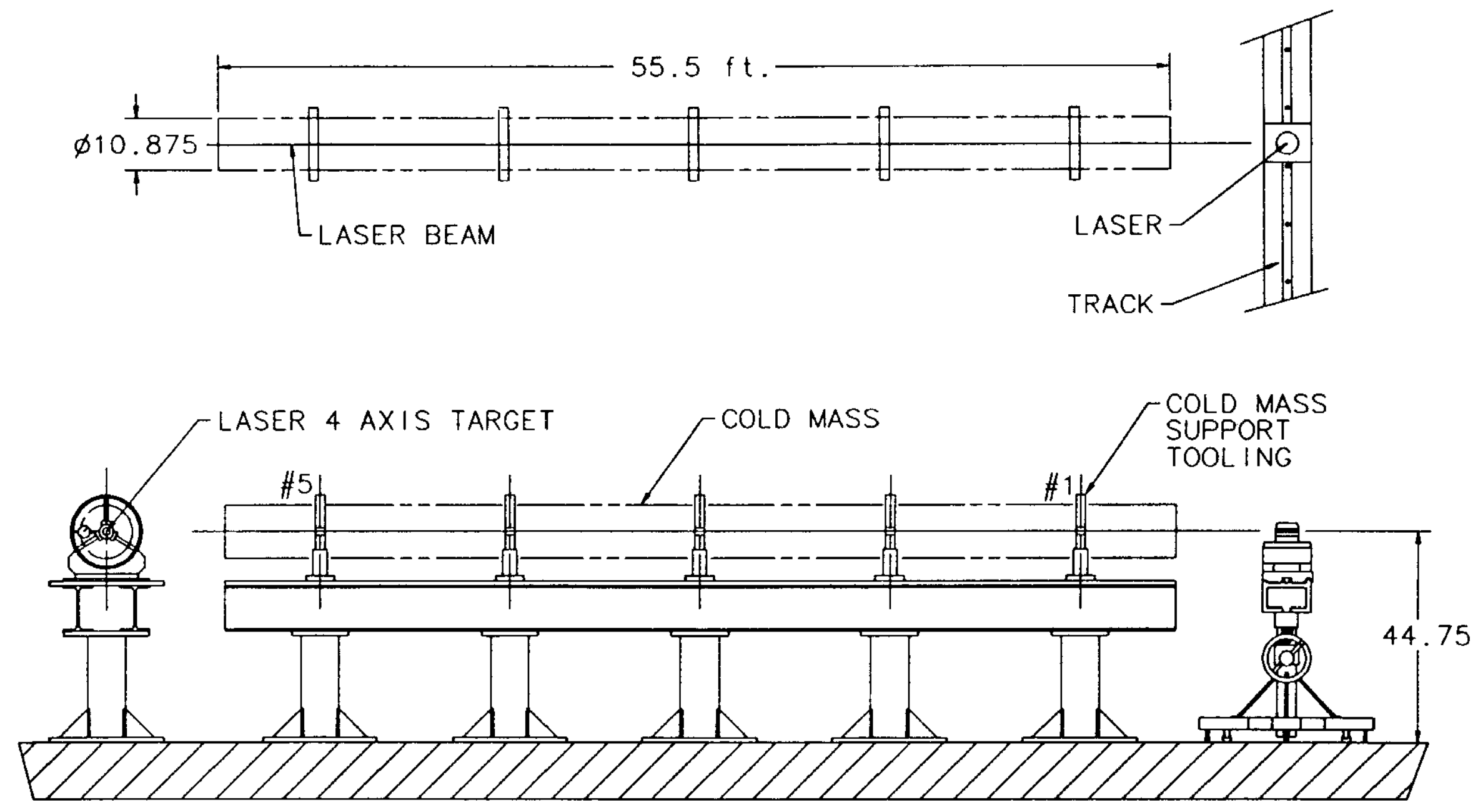

APPENDIX 1. ALIGN ALL 5 CENTERS OF SUPPORT TOOLING WITH SURVEY LINE OF SIGHT. 


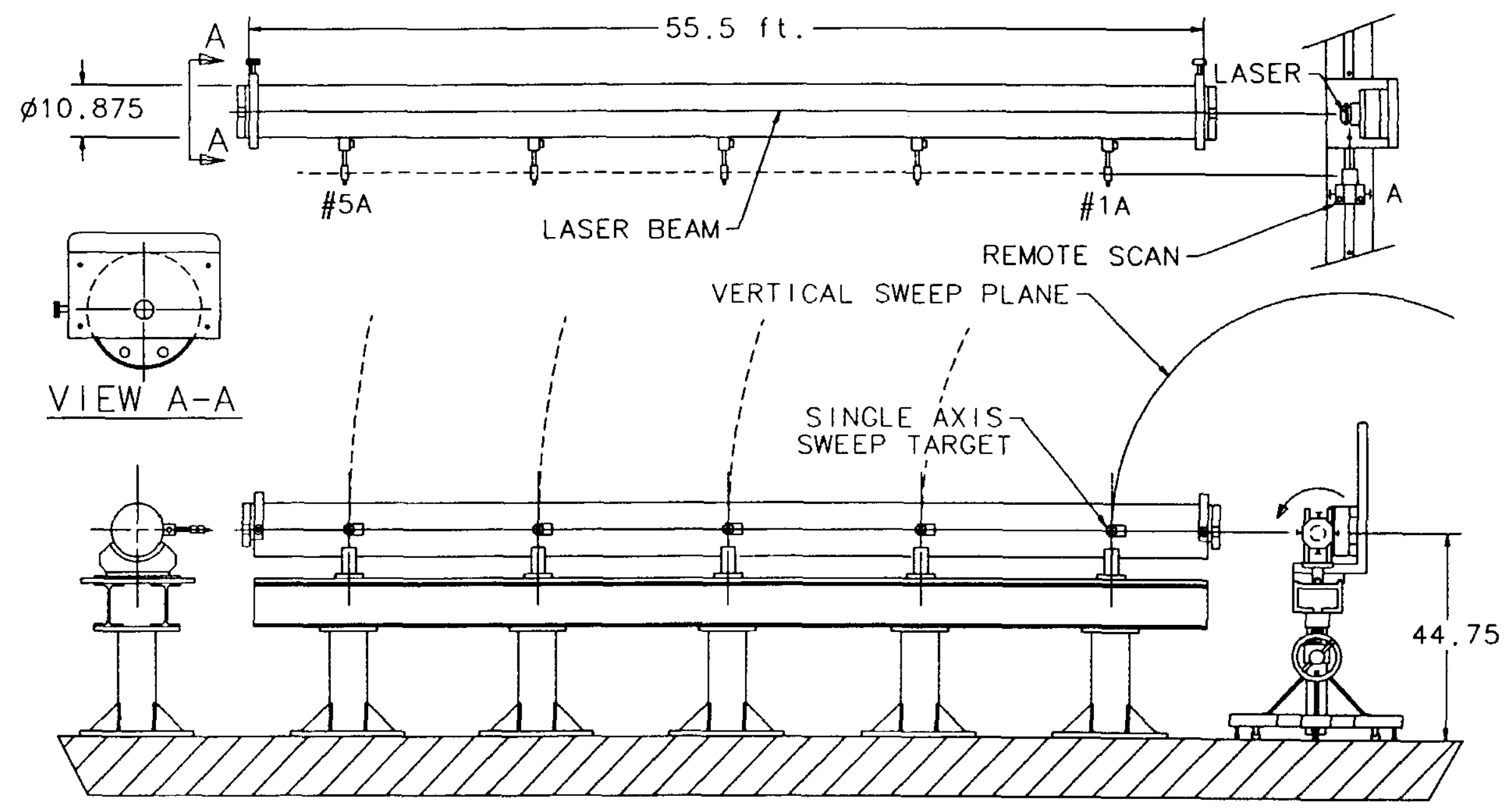

APPENDIX 2. STRAIGHTNESS OF THE COLD MASS ON THE O.D. (HORIZONTAL) 




APPENDIX 3. STRAIGHTNESS OF THE COLD MASS ON THE O.D. (VERTICAL) 


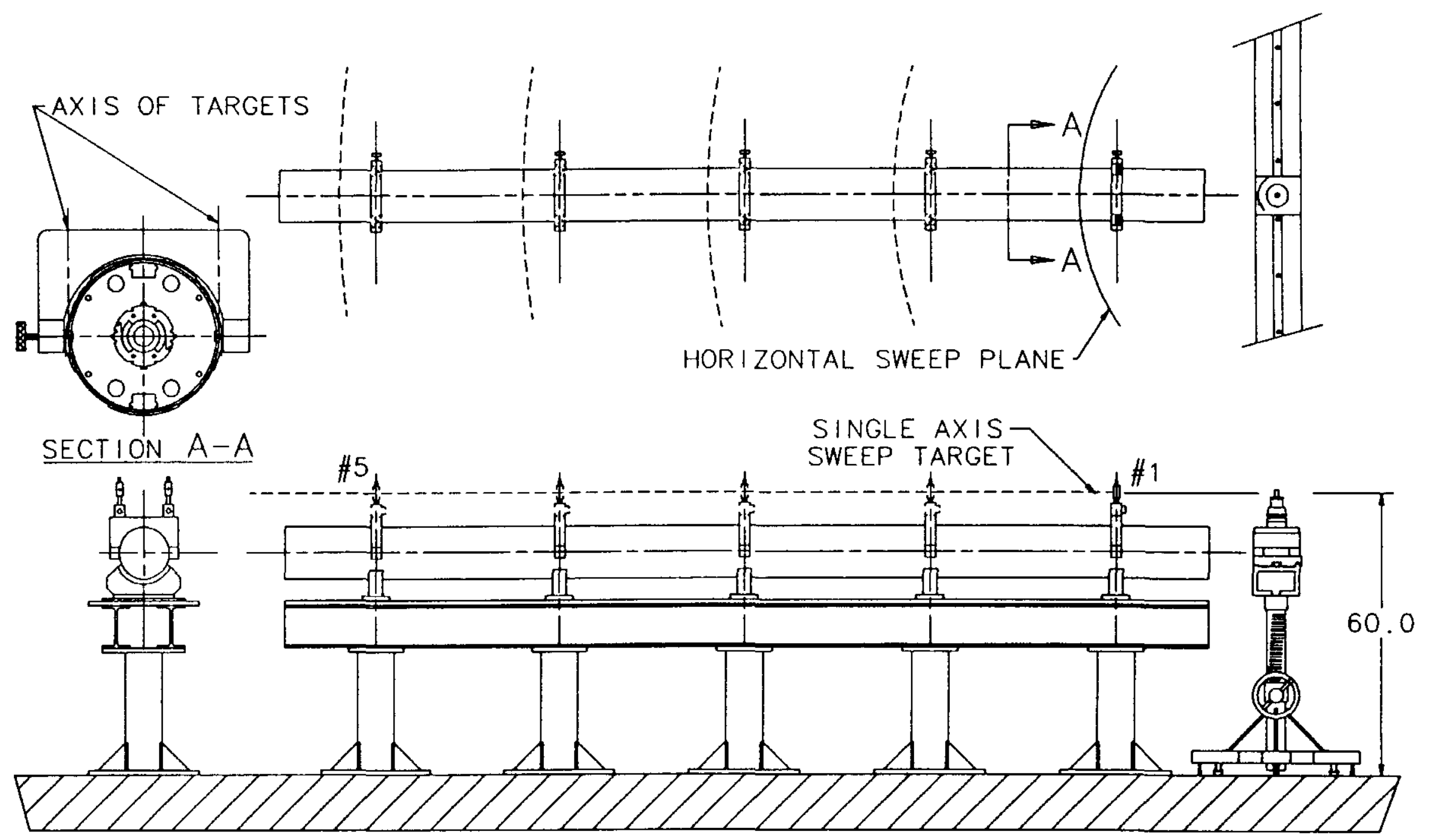

APPENDIX 4. TWIST OF COLD MASS. 

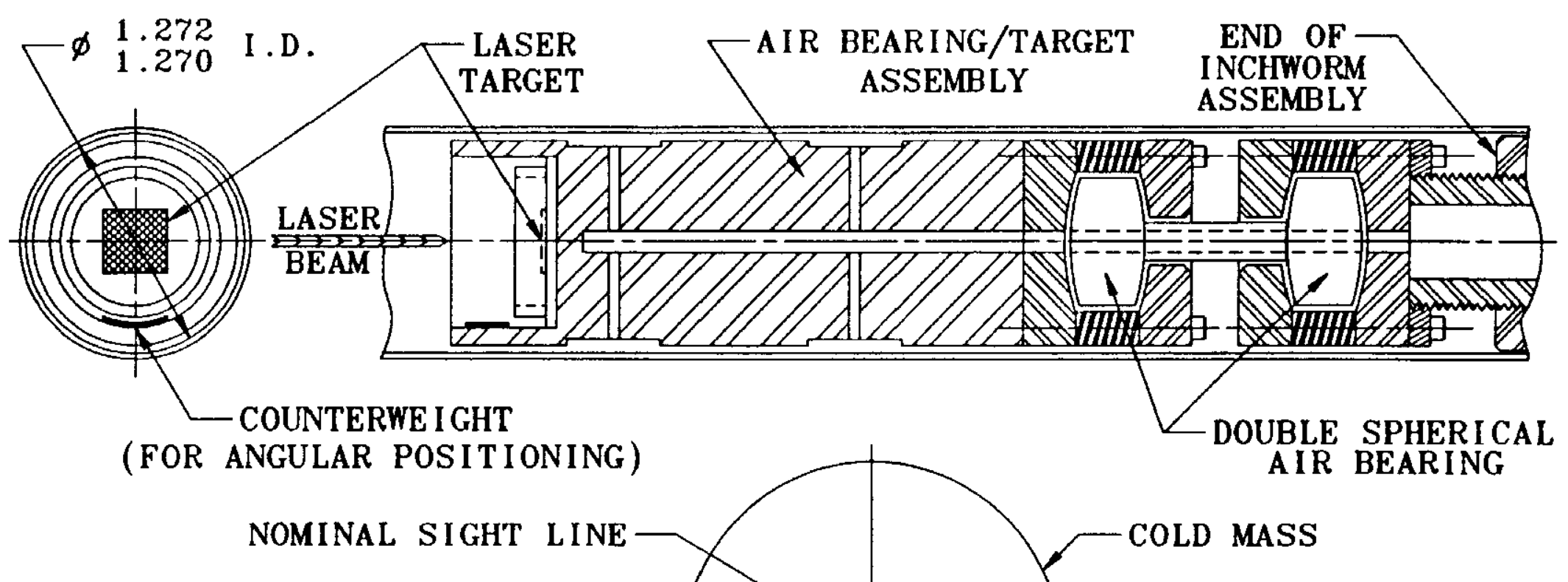

TARGET CENTER $\rightarrow-\triangle X \longrightarrow$ BEAM TUBE TARGET

APPENDIX 5. ESTABLISH REFERENCE LINE IN CENTER OF BEAM TUBE 

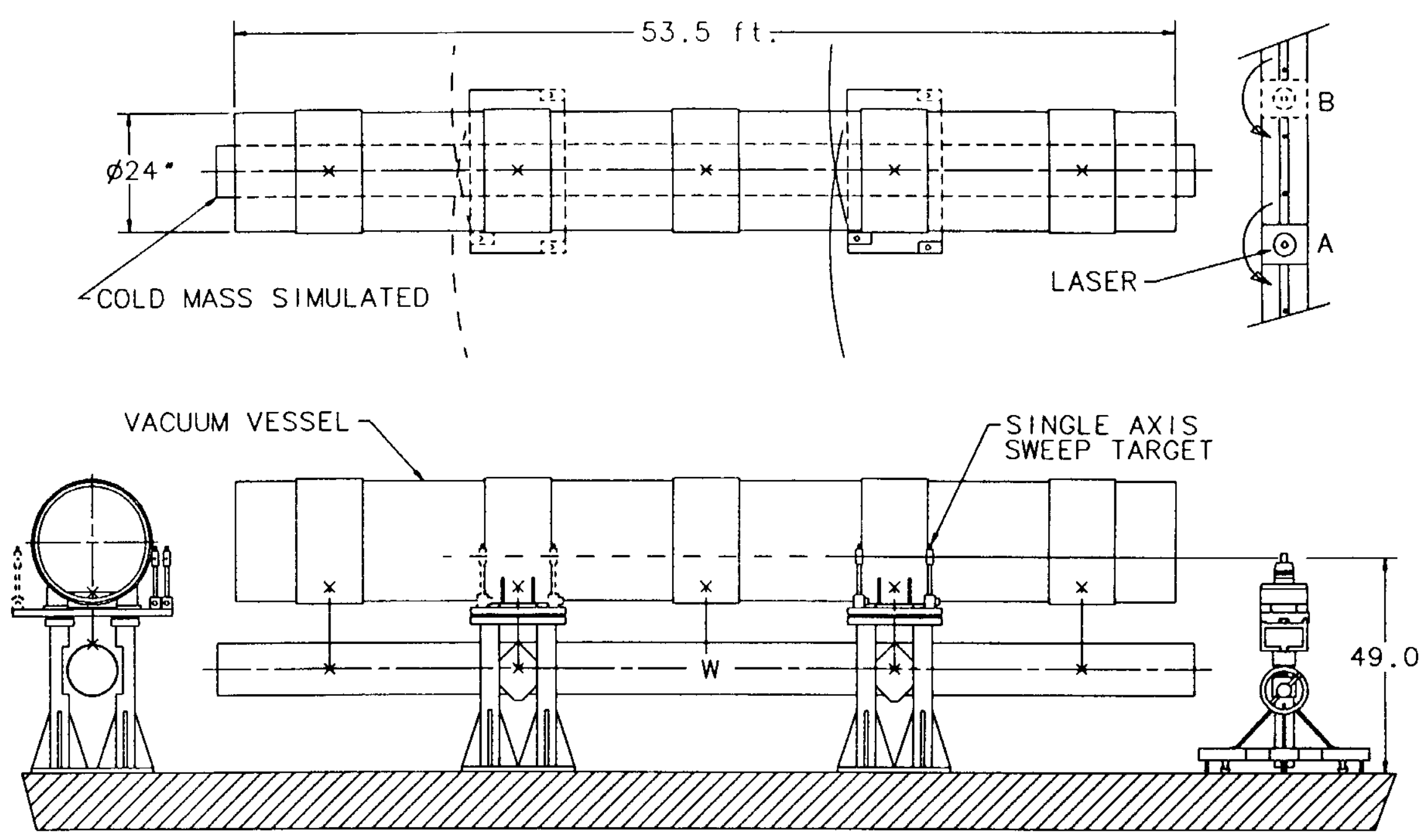

APPENDIX 6. LEVEL BOTH SUPPORT PLATFORMS . 

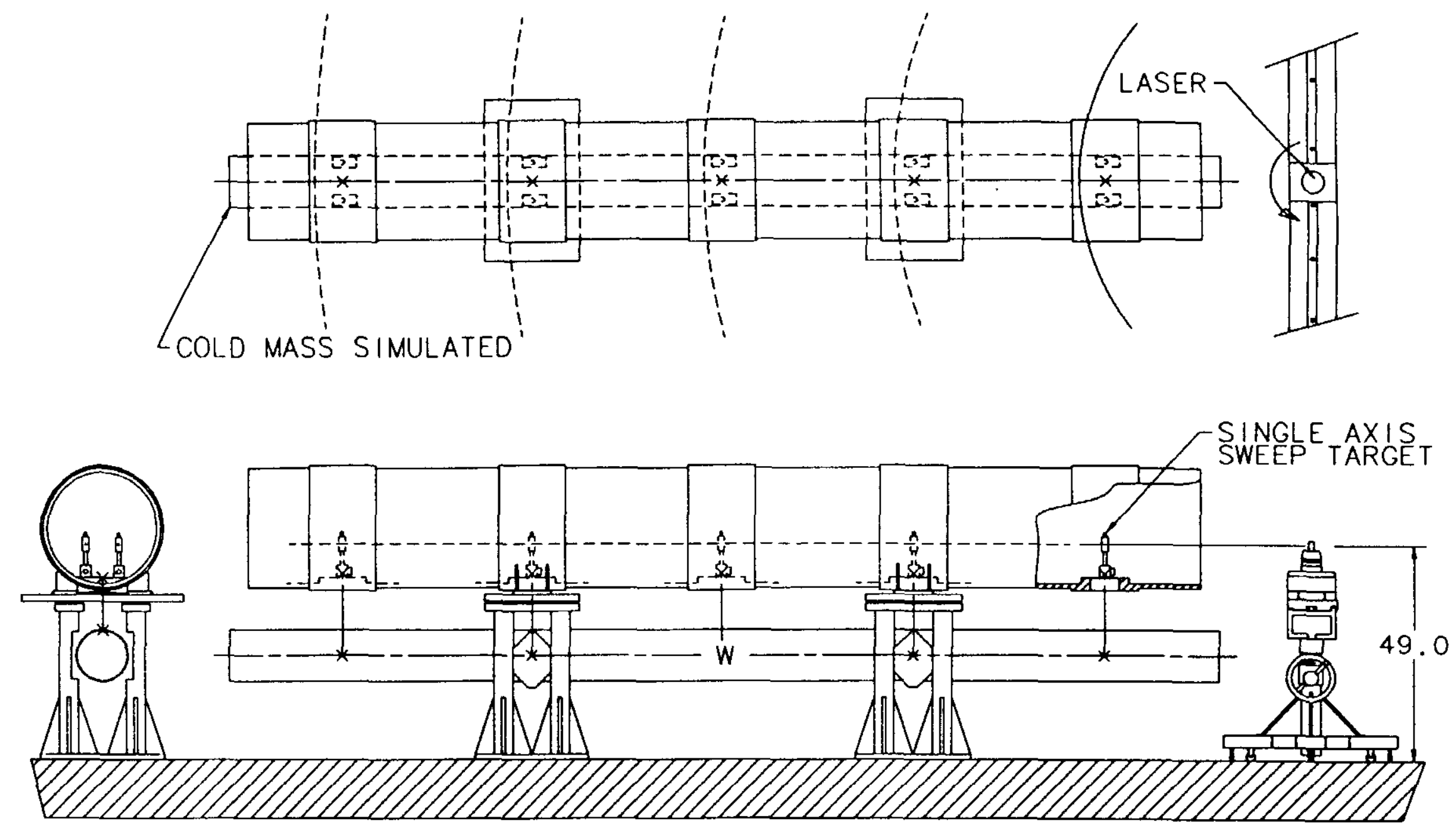
APPENDIX 7. LEVEL SURVEY OF SUPPORT SURFACES . 

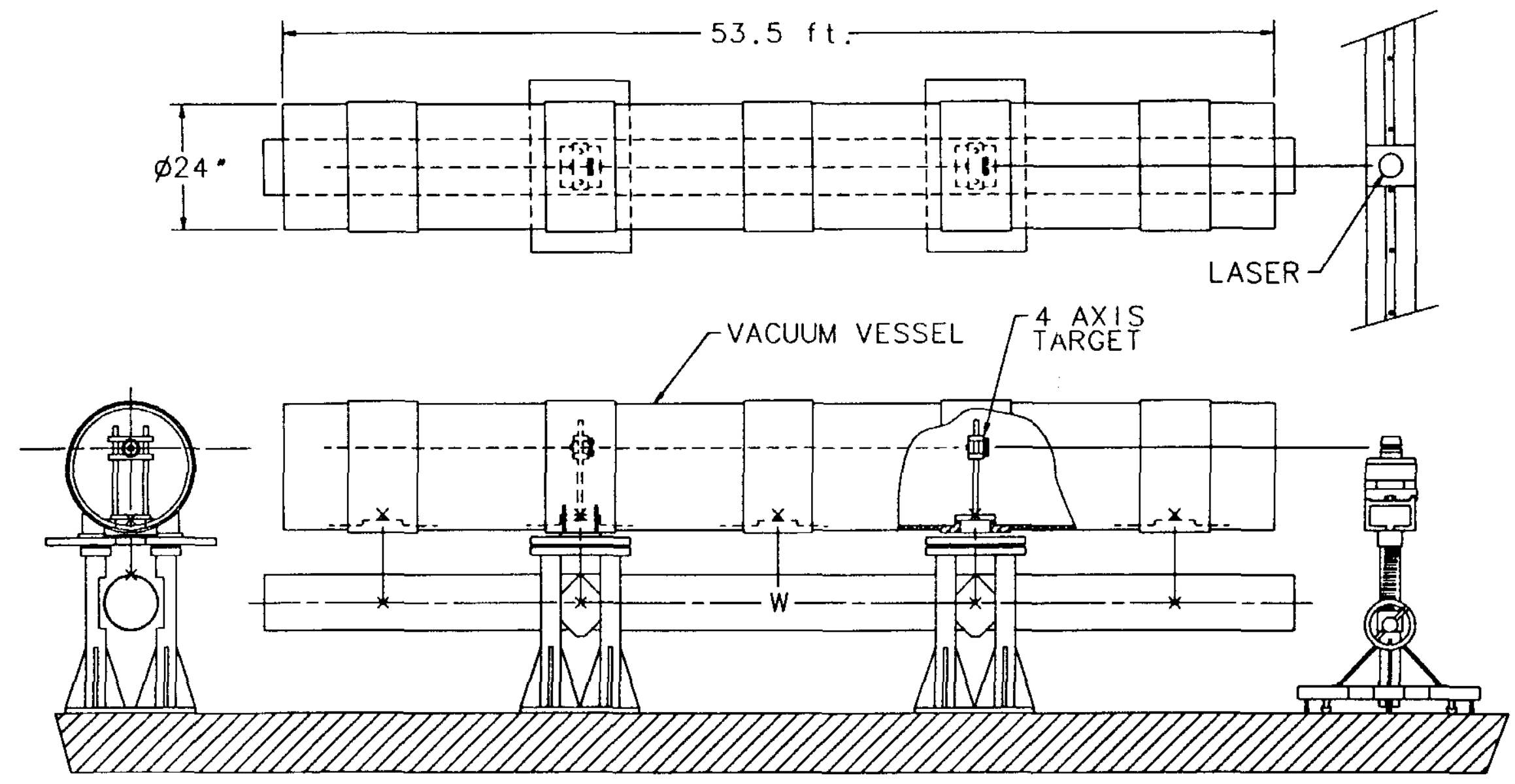

APPENDIX 8. DEF INE NOMINAL LOCATION OF COLD MASS IN VACUUM VESSEL. 


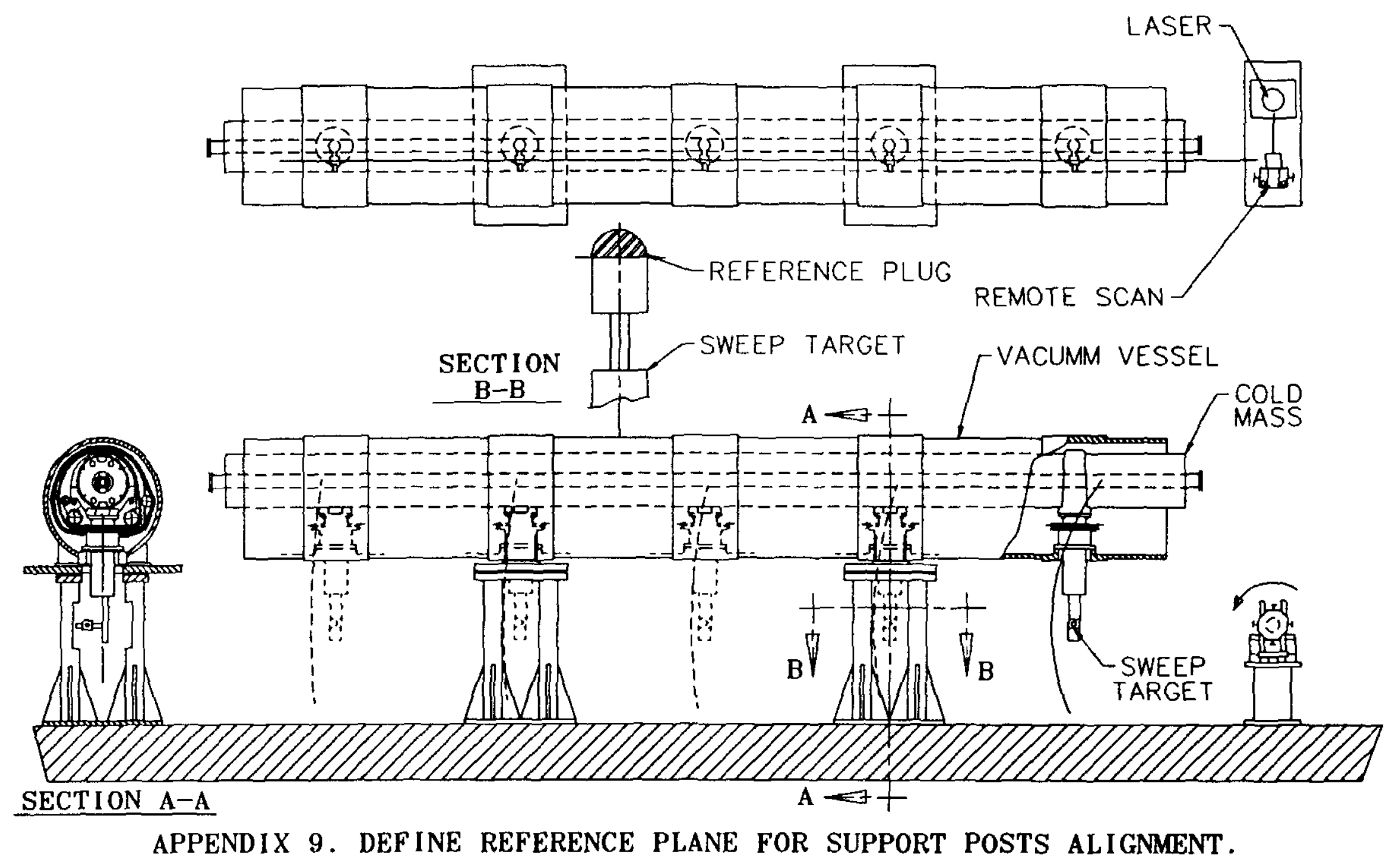




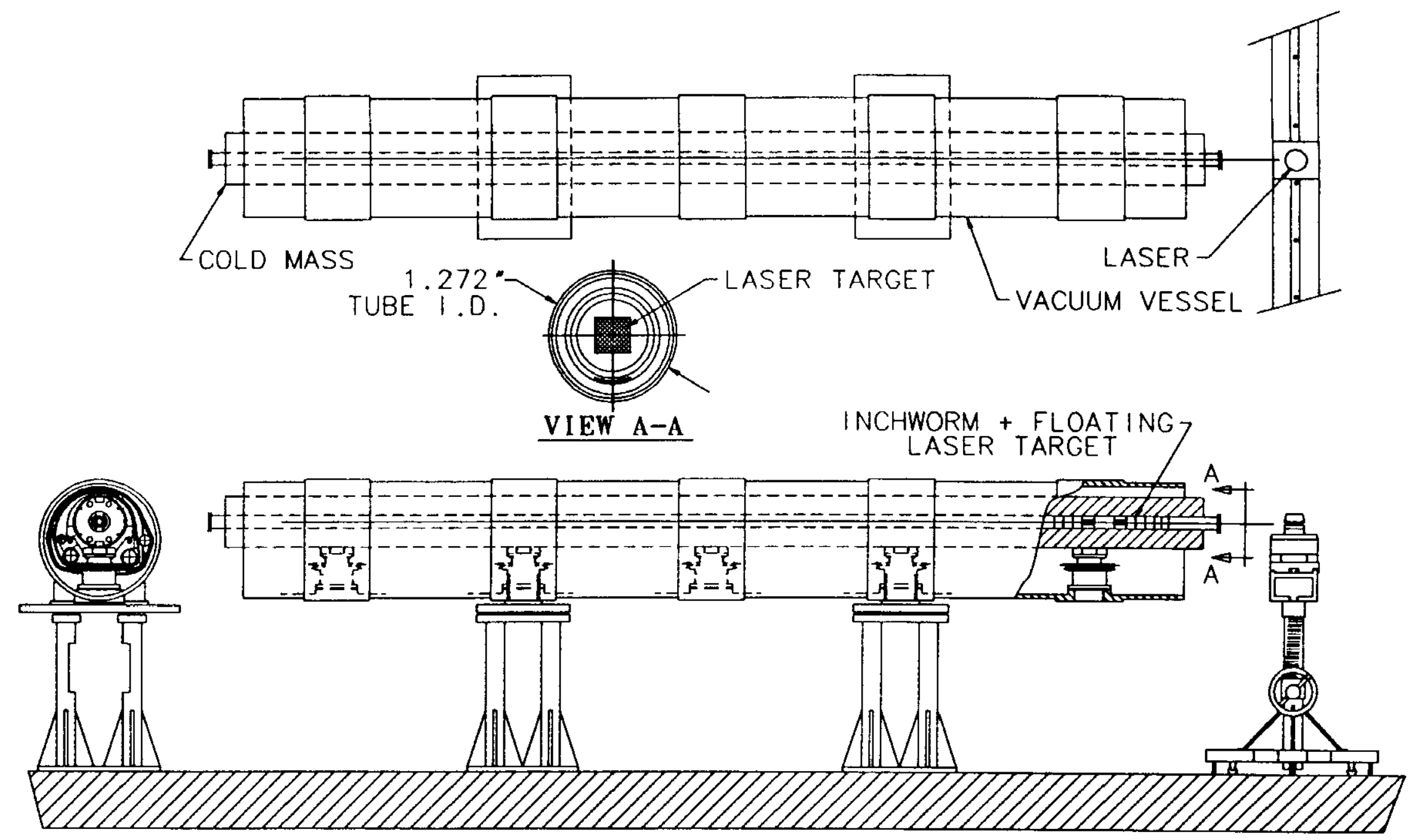

APPENDIX 10. ESTABLISH SAGITTA AND CONFIRM. 